\title{
Looking Beyond Th17 Cells: A Role for Tr1 Cells in Ankylosing Spondylitis?
}

\author{
Joanna McGinty, Nicola Brittain and Tony J. Kenna* \\ School of Biomedical Sciences, Queensland University of Technology, Brisbane, QLD, Australia
}

Keywords: ankylosing spondylitis, inflammation, T cells, regulatory T cells, Tr1 cells, genomics

\section{INTRODUCTION}

The chronic inflammatory arthritis ankylosing spondylitis (AS) is a highly heritable disease of complex genetics (1-3). The genetic association between AS and HLAB27 has been studied for almost 50 years, and over the last decade or so large-scale genomics studies have defined variants outside the $H L A$ that confer risk of developing AS $(1,2,4)$. Key among these are genes involved in $\mathrm{T}$ cell activation (including ERAP1, ERAP2, NPEPPS, UBE2E3, UBE2L3), immune signaling (including IL23R, IL6R, IL12B, TYK2) and various transcription factors involved in functional differentiation of immune cells (including TBX21, RUNX3 and EOMES). Functional genomic approaches have implicated several immune cell types in disease processes, and studies support a role for microbial dysbiosis in disease pathogenesis. It is now obvious that AS is not only a genetically complex but also immunologically complex disease. To date, however, much effort has focused on 'low hanging fruit' from genomics studies, most notably IL23R. This important work has led to advances in treatment options for AS patients through inhibiting the pathogenic effects of IL17. But trials of the IL-23 inhibitor Ustekinumab were not successful. Potential reasons for the failure of Ustekinumab Phase 3 trials in AS are many and include difficulties with outcome measures and trial design. But the failure has also sparked the community to re-evaluate subtleties in models of AS immunopathogenesis.

With many AS-associated genes implicated in various aspects of T cell biology, it is hard to pinpoint exact processes or pathways that are of critical importance in AS. Speculation needs to be supported by empirical observations from well-designed studies that push us beyond consideration of Th17 cell biology. Recently, Hanson and colleagues (5) provided evidence that AS patients exhibit significant reductions in the size of CD4 and CD8 T cell expansions globally in the peripheral blood, suggesting that perturbations in $\mathrm{T}$ cell survival, senescence, or regulation of clonal proliferation occur in AS patients during adaptive immune responses. This brings into focus what role regulatory $\mathrm{T}$ cells play in AS and indeed which populations of regulatory T cells may be of relevance to AS.

Regulatory $\mathrm{T}$ cells were originally described as a subset of immune cells critical for negative regulation of immune-mediated inflammation and prevention of autoimmune diseases. However, Tregs are also implicated in the suppression of both innate and adaptive immune cells towards allergens, organ transplants, commensals, food, and other innocuous environmental triggers (6).

FoxP $3^{+}$Tregs may be thymically induced (tTregs), peripherally induced (pTregs) or induced in cell culture, in response to TGF- $\beta$. The tissue environment promotes Tregs to express tissue-specific transcription factors that cooperate with FoxP3, providing a specialized function and supporting Treg cell subset homeostasis (7). Tregs regulate their immune environment by contact-dependent 
mechanisms, such as CD95 induction of conventional T cell apoptosis and CTLA4 downregulation of APC co-stimulatory function, as well as cytokine-mediated functions, including CD25 adsorption of IL-2 and IL-10 secretion which attenuates DC function and promotes $\operatorname{Tr} 1$ cell differentiation (8).

$\operatorname{Tr} 1$ cells, another subtype of regulatory $\mathrm{T}$ cells, do not constitutively express the transcription factor Foxp3. Upon TCR recognition of their cognate antigen at the site of tissue inflammation, Tr1 cells secrete large quantities of IL-10, which has many immunomodulatory effects on local immune cells (9). Both Tr1 and Treg cells serve a vital role in preventing deleterious immune responses with comparable mechanisms of suppression, yet Tregs are essential in the initial stage of immune suppression at the site of inflammation, while $\operatorname{Tr} 1$ cells are key for the maintenance of long-term tolerance and restoration of tissue homeostasis (10).

\section{TR1 CELLS ARE IMPORTANT REGULATORS OF INFLAMMATION}

Tr1 cells were first described in severe combined immunodeficiency disease (SCID) in the early 1990's (11). Since then, accumulating evidence implicates impaired function and reduced $\operatorname{Tr} 1$ cell numbers in immunopathogenesis of various immune-mediated diseases. Among these are inflammatory bowel disease (IBD), psoriasis, multiple sclerosis (MS), Grave's disease, Hashimoto's thyroiditis, and systemic lupus erythematosus (12-14). Broadly, IL-10 produced by Tr1 cells is a key regulator of TNF-mediated pathologies (15).

Naïve CD4+ T cells acquire a Tr1 phenotype upon cytokine signaling via IL-10, IL-21, or IL-27 which promotes STAT3 activation and subsequent priming of the IL10 locus. Transcription factors that bind to IL10 include EOMES, IRF4, c-Maf, Ahr, and Blimp-1 which act through multiple pathways to induce a stable production of IL-10 (10). In contrast, Th17 cells, although necessary for host defense against extracellular pathogens, when dysregulated become major pathogenic drivers of inflammation in many immune-mediated diseases. TGF- $\beta$ and IL- 6 are the key cytokines for initiating Th17 differentiation, which induces IL-23R expression as well as high secretion of the pro-inflammatory cytokine IL-17 $(16,17)$ (Figure 1). Microarray gene expression analysis comparing $\operatorname{Tr} 1$ cells and Th17 cells prior to IL-23 signaling identified the most predominantly overexpressed genes in $\operatorname{Tr} 1$ cells to be IRF1, IRF8, PRDM1 (Blimp-1), and TBX21 (18). IL-23 is secreted by various immune cells including dendritic cells (DCs) and macrophages in response to toll-like receptor signaling (19). Under homeostatic conditions, the presence of IL-23 in the distal small bowel promotes a localized cytokine environment that targets IL-23 sensitive intestinal cells which support mucosal barrier function and intestinal immunity. LAG-3, which is

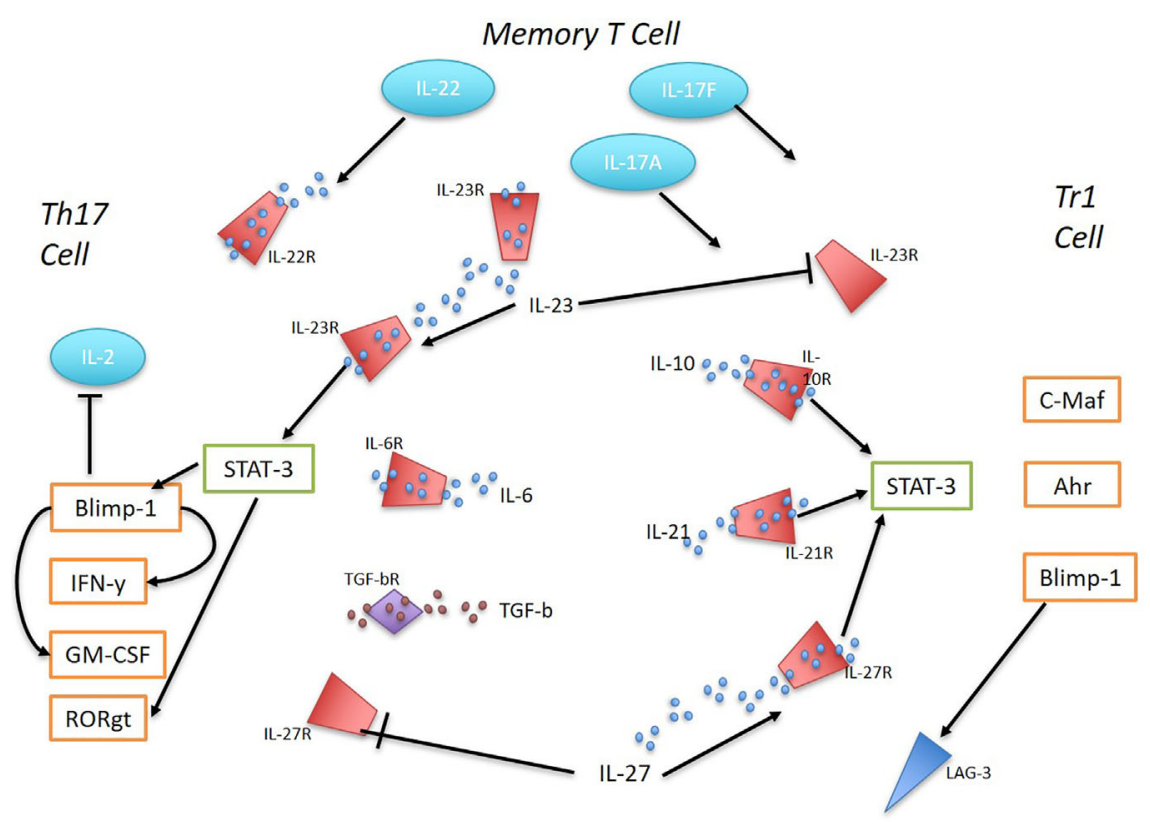

FIGURE 1 | The interplay between Th17 and Tr1 cells in inflammation. Ahr, c-Maf, and Blimp-1 promote the differentiation of Tr1 cells in response to IL-10, IL-21, and IL-27 via STAT3 activation. IL-27 was found to be sufficient to induce Blimp-1 in Tr1 cells and sustain high IL-10 production. IL-23 supresses Tr1 cells. Early Th17 cell development is initiated by TGF-B and IL-6, inducing IL-23R expression and IL-17 production. IL-23 signaling regulates Th17 cells and their pathogenicity. STAT-3 mediated IL-23-dependent Blimp-1 enhances Th17 pathogenic factors by increasing IL-23R expression, GM-CSF, IFN-y and repressing IL-2. ROR $\gamma$ t expression is stabilized by IL-23R expression. IL-27 supresses Th17 cells. Memory T cells in response to TLR signaling produce IL-23. Memory T cells with Th17 pro-inflammatory phenotype secrete large amounts of 17A, IL-17F and IL-22. 
expressed on natural regulatory $\mathrm{T}$ cells (Tregs), induced Tregs and $\operatorname{Tr} 1$ cells, has been shown to control intestinal IL-23 production by immunosuppression of CX3CR $1+$ tissueresident macrophages and innate lymphoid cells (ILCs) type 3 $(19,20)$. In vivo, IL-23R signaling suppresses the differentiation of FoxP3+ Tregs and Tr1 cells and stabilizes the expression of ROR $\mathcal{x}$, the Th17 signature transcription factor (16). IL-23 is a key factor for perpetuating and stabilizing Th17 cell activation and cytokine production as it induces strong proliferation of memory $\mathrm{T}$ cells that secrete large amounts of IL-17A, IL-17F, and IL-22 (16). IL-23-dependent signaling in Th17 cells induces Blimp-1 and in concert with T-bet, promotes pathogenicity by upregulating IL-23R expression GM-CSF and IFN-y while repressing IL-2 in a STAT3-mediated manner $(21,22)$.

Recent literature marks dysfunctional Tr1 cells and their reduced capacity to secrete large amounts of immunemediating cytokine IL-10, as attributing to persistent inflammation in autoimmune disease contexts. Tr1 dysfunction is associated with inflammation in diseases genetically and clinically relevant to AS. For example, Tr1-derived IL-10 has a non-redundant role in preventing gut inflammation in IBD (22). In a mouse model of IBD, IL23r mRNA expression is detectable on both $\operatorname{Tr} 1$ cells and Th17 cells. Tr1 cells are responsive to IL-23 and downregulate IL-10 in response to IL-23R signaling (22). Clinical and genetic overlap between AS and IBD has been recognized for many years, and an increase in IL-23 has been well documented in both diseases $(23,24)$. Psoriasis, frequently concomitant with AS, is driven by chronic activation of autoreactive Th17 cells (25). Psoriasis patients exhibit an inverse relationship between disease severity and $\operatorname{Tr} 1$ and Treg cell numbers. Tr1 cells were not found in the skin of healthy controls; however, $\operatorname{Tr} 1$ cells were identified in the nonlesioned skin of psoriasis patients. Psoriatic lesions revealed an increase in activated $\mathrm{CD}^{+}{ }^{+} \mathrm{CD} 4^{+} \mathrm{CD} 69^{+} \mathrm{T}$ cells and a lack of $\mathrm{Tr} 1$ cells (26).

\section{THE POTENTIAL ROLE OF THE IL-12/IL-23 AND IL-27 AXIS IN AS IMMUNOPATHOLOGY}

IL-27 signaling induces Blimp-1-mediated IL-10 production in Tr1 cells, and in the absence of IL-23 signaling, Th17 cells respond to IL27 and IL-12 signaling by secreting IL-10 in a Blimp-1 dependent manner. This demonstrates a potential for plasticity in Th17 cells as they lose their pathogenicity and adopt a Tr1-like phenotype which can contribute to homeostasis under certain conditions (18). In contrast, Th17 cells further stimulated by IL-23 demonstrated a commitment to the inflammatory phenotype (27), known to be implicated in many autoinflammatory conditions including AS (23). IL-27 levels are reported to be elevated in AS patients and to correlate with disease activity measures (28) which would seem to support development of Tr1 cells in AS patients. However, IL-23 counteracts IL-27 and IL-12-mediated effects on Tr1-development reinforcing the pro-inflammatory phenotype of Th17 cells (18). The balance between IL-23 vs IL-12/IL-27 signaling in CD4+ effector T cells determines whether tissue inflammation is perpetuated or resolved. It is our opinion that the immunomodulatory function of regulatory T cells is impaired in the context of AS, resulting in perpetual inflammation in the enthesis and ileum of patients with active disease. It is hypothesized that deficient IL-27 signaling, reduced IL-10 production by Tr1 cells and exacerbated IL-23 signaling promotes persistent IL-17 and other pro-inflammatory cytokine production and proliferation of pro-inflammatory $\mathrm{T}$ cell subsets. These three key factors that may diminish the capacity for immune regulation are linked to known AS genetic risk factors including IL27, IL23R, TBX21, and EOMES susceptibility mutations $(1,2)$. However, the link between AS susceptibility loci and development of Trl cells in AS has been further complicated recently. Pepelyayeva and colleagues reported reduced numbers of Tr1 cells in Erap-/- mice (29), a genotype that GWAS data associates with AS protection rather than risk (1). A better understanding of the role, if any, $\operatorname{Tr} 1$ cells play in AS patients may be a valuable step towards understanding how to control inflammation in this disease.

\section{CAN TR1 CELLS OFFER ANY THERAPEUTIC POTENTIAL IN AS?}

It is clear that $\operatorname{Tr} 1$ cells are an important regulator of general immune responses. Therapeutic manipulation of $\operatorname{Tr} 1$ cells, ex vivo or in vivo might be highly advantageous in several $\mathrm{T}$ cellmediated diseases. Much progress has already been made in animal models, which proved that $\operatorname{Tr} 1$ cell-based therapies may be a feasible approach to treating inflammatory disorders in general.

Adoptive transfer of in vitro induced Ag-specific Tr1 cells efficiently prevents colitis induced in SCID mice by pathogenic $\mathrm{T}$ cells (30). In a pre-clinical model of type 1 diabetes $\operatorname{Tr} 1$ cells induced in the intestinal mucosa migrate to the periphery and control effector $\mathrm{T}$ cell responses and the development of diabetes (31). Studies in MS attributed deficiencies in IL-10-secreting Tr1 cells to decreased IL-27 and disruption of the CD46 pathway that promotes transformation of IFN-y-secreting Th1 cells into $\operatorname{Tr} 1$ cells. Exogenous IL-27 partially restored the number and function in a mouse model of MS, experimental autoimmune encephalomyelitis (EAE) (32). IL-27 induced Tr1 cells have been described to have therapeutic potential in several autoimmune contexts by expanding their immunomodulatory function in active disease states.

Robust protocols have been established to generate clinicalgrade human $\operatorname{Tr} 1$ cells $(33,34)$ and $\operatorname{Tr} 1$ cell-based therapies have been trialed in graft versus host disease (35) and refractory Crohn's disease (36) clinical trials.

\section{DISCUSSION}

$\operatorname{Tr} 1$ cells have an important role in autoimmune disease prevention, and investigating their potential to restore immune homeostasis in environments of persistent inflammation may be 
beneficial in the context of AS. While the genetics of AS implicate $\operatorname{Tr} 1$ biology in disease processes discrepancies exist between in vitro data that suggest the cytokine environment in AS may be suitable for expansion of $\operatorname{Tr} 1$ cells (28) and in vivo data that show reduced $\operatorname{Tr} 1$ cells in mice that lack an important ASsusceptibility gene (29). A challenge from here is to define where and how Tr1 cells may be important in AS and to define pre-clinical models that will allow pre-clinical evaluation of their therapeutic potential in AS.

\section{REFERENCES}

1. Evans DM, Spencer CC, Pointon JJ, Su Z, Harvey D, Kochan G, et al. Interaction between ERAP1 and HLA-B27 in ankylosing spondylitis implicates peptide handling in the mechanism for HLA-B27 in disease susceptibility. Nat Genet (2011) 43(8):761-7. doi: 10.1038/ng0911-919a

2. International Genetics of Ankylosing Spondylitis Consortium, Cortes A, Hadler J, Pointon JP, Robinson PC, Karaderi T, et al. Identification of multiple risk variants for ankylosing spondylitis through high-density genotyping of immune-related loci. Nat Genet (2013) 45(7):730-8. doi: $10.1038 /$ ng.2667

3. Brown MA, Kenna T, Wordsworth BP. Genetics of ankylosing spondylitisinsights into pathogenesis. Nat Rev Rheumatol (2016) 12(2):81-91. doi: 10.1038/nrrheum.2015.133

4. Ellinghaus D, Jostins L, Spain SL, Cortes A, Bethune J, Han B, et al. Analysis of five chronic inflammatory diseases identifies 27 new associations and highlights disease-specific patterns at shared loci. Nat Genet (2016) 48:5108. doi: $10.1038 / \mathrm{ng} .3528$

5. Hanson AL, Nel HJ, Bradbury L, Phipps J, Thomas R, Le Cao KA, et al. T-cell receptor immunosequencing reveals altered repertoire diversity and diseaseassociated clonal expansions in ankylosing spondylitis patients. Arthritis Rheumatol (2020) 72(8):1289-302. doi: 10.1002/art.41252

6. Okeke EB, Uzonna JE. The Pivotal Role of Regulatory T Cells in the Regulation of Innate Immune Cells. Front Immunol (2019) 10:680. doi: 10.3389/fimmu.2019.00680

7. Josefowicz SZ, Lu LF, Rudensky AY. Regulatory T cells: mechanisms of differentiation and function. Annu Rev Immunol (2012) 30:531-64. doi: 10.1146/annurev.immunol.25.022106.141623

8. Sakaguchi S, Miyara M, Costantino CM, Hafler DA. FOXP3+ regulatory T cells in the human immune system. Nat Rev Immunol (2010) 10(7):490-500. doi: $10.1038 /$ nri2785

9. Gregori S, Roncarolo MG. Engineered T Regulatory Type 1 Cells for Clinical Application. Front Immunol (2018) 9:233. doi: 10.3389/fimmu.2018.00233

10. Roncarolo MG, Gregori S, Bacchetta R, Battaglia M, Gagliani N. The Biology of T Regulatory Type 1 Cells and Their Therapeutic Application in ImmuneMediated Diseases. Immunity (2018) 49(6):1004-19. doi: 10.1016/ j.immuni.2018.12.001

11. Bacchetta R, Bigler M, Touraine JL, Parkman R, Tovo PA, Abrams J, et al. High levels of interleukin 10 production in vivo are associated with tolerance in SCID patients transplanted with HLA mismatched hematopoietic stem cells. J Exp Med (1994) 179(2):493-502. doi: 10.1084/jem.179.2.493

12. Jia X, Zhai T, Wang B, Yao Q, Li Q, Mu K, et al. Decreased number and impaired function of type 1 regulatory T cells in autoimmune diseases. J Cell Physiol (2019) 234(8):12442-50. doi: 10.1002/jcp.28092

13. Vitales-Noyola M, Serrano-Somavilla A, Martinez-Hernandez R, SampedroNunez M, Ramos-Levi AM, Gonzalez-Amaro R, et al. Patients With Autoimmune Thyroiditis Show Diminished Levels and Defective Suppressive Function of Tr1 Regulatory Lymphocytes. J Clin Endocrinol Metab (2018) 103(9):3359-67. doi: 10.1210/jc.2018-00498

14. Le Buanec H, Gougeon ML, Mathian A, Lebon P, Dupont JM, Peltre G, et al. IFN-alpha and CD46 stimulation are associated with active lupus and skew natural $\mathrm{T}$ regulatory cell differentiation to type 1 regulatory $\mathrm{T}(\mathrm{Tr} 1)$ cells. Proc Natl Acad Sci USA (2011) 108(47):18995-9000. doi: 10.1073/pnas. 1113301108

\section{AUTHOR CONTRIBUTIONS}

All authors contributed to the article and approved the submitted version.

\section{FUNDING}

TK is supported by an NHMRC Project grant (APP1162767).

15. Montes de Oca M, Kumar R, de Labastida Rivera F, Amante FH, Sheel M, Faleiro RJ, et al. Blimp-1-Dependent IL-10 Production by Tr1 Cells Regulates TNF-Mediated Tissue Pathology. PLoS Pathog (2016) 12(1):e1005398. doi: 10.1371/journal.ppat.1005398

16. Jain R, Chen Y, Kanno Y, Joyce-Shaikh B, Vahedi G, Hirahara K, et al. Interleukin-23-Induced Transcription Factor Blimp-1 Promotes Pathogenicity of T Helper 17 Cells. Immunity (2016) 44(1):131-42. doi: 10.1016/j.immuni.2015.11.009

17. Wilke CM, Bishop K, Fox D, Zou W. Deciphering the role of Th17 cells in human disease. Trends Immunol (2011) 32(12):603-11. doi: 10.1016/ j.it.2011.08.003

18. Heinemann C, Heink S, Petermann F, Vasanthakumar A, Rothhammer V, Doorduijn E, et al. IL-27 and IL-12 oppose pro-inflammatory IL-23 in CD4+ T cells by inducing Blimp1. Nat Commun (2014) 5:3770. doi: 10.1038/ncomms4770

19. Neurath MF. IL-23 in inflammatory bowel diseases and colon cancer. Cytokine Growth Factor Rev (2019) 45:1-8. doi: 10.1016/j.cytogfr.2018.12.002

20. Zhao H, Liao X, Kang Y. Tregs: Where We Are and What Comes Next? Front Immunol (2017) 8:1578. doi: 10.3389/fimmu.2017.01578

21. Bastian D, Wu Y, Betts BC, Yu XZ. The IL-12 Cytokine and Receptor Family in Graft-vs.-Host Disease. Front Immunol (2019) 10:988. doi: 10.3389/ fimmu.2019.00988

22. Alfen JS, Larghi P, Facciotti F, Gagliani N, Bosotti R, Paroni M, et al. Intestinal IFN-gamma-producing type 1 regulatory $\mathrm{T}$ cells coexpress CCR5 and programmed cell death protein 1 and downregulate IL-10 in the inflamed guts of patients with inflammatory bowel disease. J Allergy Clin Immunol (2018) 142(5):1537-47 e8. doi: 10.1016/j.jaci.2017.12.984

23. Brown MA, Kenna T, Wordsworth BP. Genetics of ankylosing spondylitisinsights into pathogenesis. Nat Rev Rheumatol (2016) 12(2):81-91. doi: 10.1038/nrrheum.2015.133

24. Ciccia F, Bombardieri M, Principato A, Giardina A, Tripodo C, Porcasi R, et al. Overexpression of interleukin-23, but not interleukin-17, as an immunologic signature of subclinical intestinal inflammation in ankylosing spondylitis. Arthritis Rheum (2009) 60(4):955-65. doi: 10.1002/art.24389

25. Zaba LC, Fuentes-Duculan J, Eungdamrong NJ, Abello MV, Novitskaya I, Pierson KC, et al. Psoriasis is characterized by accumulation of immunostimulatory and Th1/Th17 cell-polarizing myeloid dendritic cells. J Invest Dermatol (2009) 129(1):79-88. doi: 10.1038/jid.2008.194

26. Kim J, Lee J, Gonzalez J, Fuentes-Duculan J, Garcet S, Krueger JG. Proportion of CD4(+)CD49b(+)LAG-3(+) Type 1 Regulatory T Cells in the Blood of Psoriasis Patients Inversely Correlates with Psoriasis Area and Severity Index. J Invest Dermatol (2018) 138(12):2669-72. doi: 10.1016/j.jid.2018.05.021

27. Stritesky GL, Yeh N, Kaplan MH. IL-23 promotes maintenance but not commitment to the Th17 lineage. J Immunol (2008) 181(9):5948-55. doi: 10.4049/jimmunol.181.9.5948

28. Lin TT, Lu J, Qi CY, Yuan L, Li XL, Xia LP, et al. Elevated serum level of IL-27 and VEGF in patients with ankylosing spondylitis and associate with disease activity. Clin Exp Med (2015) 15(2):227-31. doi: 10.1007/s10238-014-0281-x

29. Pepelyayeva Y, Rastall DPW, Aldhamen YA, O'Connell P, Raehtz S, Alyaqoub FS, et al. ERAP1 deficient mice have reduced Type 1 regulatory $\mathrm{T}$ cells and develop skeletal and intestinal features of Ankylosing Spondylitis. Sci Rep (2018) 8(1):12464. doi: 10.1038/s41598-018-30159-5

30. Groux H, O’Garra A, Bigler M, Rouleau M, Antonenko S, de Vries JE, et al. A CD4+ T-cell subset inhibits antigen-specific T-cell responses and prevents colitis. Nature (1997) 389(6652):737-42. doi: 10.1038/39614 
31. Yu H, Gagliani N, Ishigame H, Huber S, Zhu S, Esplugues E, et al. Intestinal type 1 regulatory $\mathrm{T}$ cells migrate to periphery to suppress diabetogenic $\mathrm{T}$ cells and prevent diabetes development. Proc Natl Acad Sci USA (2017) 114 (39):10443-8. doi: 10.1073/pnas.1705599114

32. Meka RR, Venkatesha SH, Dudics S, Acharya B, Moudgil KD. IL-27-induced modulation of autoimmunity and its therapeutic potential. Autoimmun Rev (2015) 14(12):1131-41. doi: 10.1016/j.autrev.2015.08.001

33. Mfarrej B, Tresoldi E, Stabilini A, Paganelli A, Caldara R, Secchi A, et al. Generation of donor-specific Trl cells to be used after kidney transplantation and definition of the timing of their in vivo infusion in the presence of immunosuppression. J Transl Med (2017) 15(1):40. doi: 10.1186/s12967-017-1133-8

34. Bacchetta R, Gregori S, Serafini G, Sartirana C, Schulz U, Zino E, et al. Molecular and functional characterization of allogantigen-specific anergic $\mathrm{T}$ cells suitable for cell therapy. Haematologica (2010) 95(12):2134-43. doi: 10.3324/haematol.2010.025825

35. Bacchetta R, Lucarelli B, Sartirana C, Gregori S, Lupo Stanghellini MT, Miqueu $\mathrm{P}$, et al. Immunological Outcome in Haploidentical-HSC
Transplanted Patients Treated with IL-10-Anergized Donor T Cells. Front Immunol (2014) 5:16. doi: 10.3389/fimmu.2014.00016

36. Desreumaux P, Foussat A, Allez M, Beaugerie L, Hebuterne X, Bouhnik Y, et al. Safety and efficacy of antigen-specific regulatory T-cell therapy for patients with refractory Crohn's disease. Gastroenterology (2012) 143 (5):1207-17 e2. doi: 10.1053/j.gastro.2012.07.116

Conflict of Interest: The authors declare that the research was conducted in the absence of any commercial or financial relationships that could be construed as a potential conflict of interest.

Copyright (c) 2020 McGinty, Brittain and Kenna. This is an open-access article distributed under the terms of the Creative Commons Attribution License (CC BY). The use, distribution or reproduction in other forums is permitted, provided the original author(s) and the copyright owner(s) are credited and that the original publication in this journal is cited, in accordance with accepted academic practice. No use, distribution or reproduction is permitted which does not comply with these terms. 